\title{
Aberrant expression of B7-H4 may contribute to the development of hepatocellular carcinoma
}

\author{
LINGLING YUAN $^{1 *}$, LIJIE DONG $^{1^{*}}$, GUOHUA YU $^{2^{*}}$, WANFENG FAN ${ }^{1}$, \\ LIN ZHANG $^{1}$, PEIYUAN WANG ${ }^{1}$, XUEMEI HU ${ }^{3}$ and MINGDONG ZHAO ${ }^{1}$ \\ ${ }^{1}$ Department of Radiology, Binzhou Affiliated Hospital of Binzhou Medical University, Binzhou, \\ Shandong 256603; ${ }^{2}$ Department of Pathology, Yu Huang Ding Hospital, Yantai, Shandong 264000; \\ ${ }^{3}$ Department of Immunology, Binzhou Medical University, Yantai, Shandong 264003, P.R. China
}

Received August 19, 2015; Accepted September 16, 2016

DOI: $10.3892 / \mathrm{mmr} .2016 .5887$

\begin{abstract}
In order to determine the effect of B7-H4 on the development of human hepatocellular carcinoma (HCC), the expression levels of B7-H4 were evaluated using reverse transcription-polymerase chain reaction and flow cytometry in HL-7702 and Huh7 cells. B7-H4 protein expression levels were analyzed using western blotting and immunohistochemistry in $\mathrm{HCC}$ tissues collected from patients and from a mouse tumor model. Soluble B7-H4 (sB7-H4), interferon- $\gamma($ IFN- $\gamma$ ), and interleukin-4 (IL-4) in blood serum were assessed using ELISA in patients with $\mathrm{HCC}$ and mice injected with tumor cells. B7-H4 was expressed in HCC cell lines, mouse tumor tissues and HCC patient tissues. However, B7-H4 was not detected in HL-7702 cells or normal human liver tissues. The expression level of B7-H4 was positively correlated with tumor-node-metastasis (TNM) stage, lymph node metastasis, and differentiation degree in patients with HCC. sB7-H4 levels in blood serum samples collected from patients with HCC and tumorigenic mice were higher compared with healthy controls. Expression levels of IFN- $\gamma$ were reduced, and IL-4 levels were increased in blood serum samples of patients with $\mathrm{HCC}$ and tumorigenic mice compared with healthy controls. sB7-H4 expression levels were negatively correlated with IFN- $\gamma$ levels, and with the ratio of IFN- $\gamma$ to IL-4. Additionally, sB7-H4 was positively correlated with IL-4 levels in mouse tumor tissues,
\end{abstract}

Correspondence to: Dr Mingdong Zhao, Department of Radiology, Binzhou Affiliated Hospital of Binzhou Medical University, 661 Huang-He Road, Binzhou, Shandong 256603, P.R. China

E-mail: mingdongzhao@126.com

Dr Xuemei Hu, Department of Immunology, Binzhou Medical University, 346 Lai-Shan Road, Yantai, Shandong 264003, P.R. China

E-mail: xue-mei-hu@163.com

*Contributed equally

Key words: B7-H4, sB7-H4, aberrant expression, pathogenesis, hepatocellular carcinoma serum samples obtained from tumorigenic mice and human HCC patients. Notably, the levels of sB7-H4 and IL-4 were positively correlated and IFN- $\gamma$ was negatively correlated with the TNM stage of patients with HCC. In addition, sB7-H4 and IL-4 expression levels increased and levels of IFN- $\gamma$ and the ratio of IFN- $\gamma / \mathrm{IL}-4$ decreased as a function of time post tumor implantation in the mouse model. The present study determined that aberrant expression of $\mathrm{B} 7-\mathrm{H} 4$ contributed to $\mathrm{HCC}$ development. B7-H4 may be a potential target for therapy and diagnosis of HCC.

\section{Introduction}

Hepatocellular carcinoma (HCC) is the fifth most common cancer in the world accounting for $\sim 600,000$ mortalities each year (1-3). HCC is the third most common cause of cancer mortality and is more prevalent in developing countries, particularly those located in Eastern and South-Eastern Asia compared with the developed world $(4,5)$. In China, the age-standardized incidence rate of $\mathrm{HCC}$ is 37.9 per 100,000 for men and 14.2 per 100,000 for women (6). Tumor resection, liver transplantation, radiofrequency (thermal) ablation, percutaneous ethanol injection and transarterial chemoembolization are the primary treatment options (7-9); however, these treatments are expensive. Therefore, additional investigation of the pathogenesis of HCC with the goal of identifying effective and affordable methods of treatment is required.

The B7 family of T cell co-stimulatory and co-inhibitory molecules is vital for the regulation of adaptive immune responses. B7-H4 (also termed B7x or B7S1) has been determined to be involved in the downregulation of antigen-specific immune responses as it inhibited T cell proliferation, cell cycle progression, and cytokine production (10-12). In mice, B7-H4 transcripts were ubiquitously expressed; however, no protein expression was detected (11-13). A previous study did not detect B7-H4 protein expression in normal human tissues (14). However, B7-H4 has been overexpressed in various human tumors, including esophageal squamous cell carcinoma (15), pancreatic cancer $(16)$, gastric cancer $(17,18)$, colorectal carcinoma (19) and lung cancer (20). A previous study also reported that B7-H4 is expressed in patients with liver cancer (21); 
however, whether the level of B7-H4 may be correlated with HCC pathogenesis remains to be elucidated.

B7-H4 may inhibit the function of human T cells (22). Th1 and Th2 $\mathrm{CD}^{+} \mathrm{T}$ cells are crucial for effective immune protection (23). Th1 cells mediate antitumor reactivity through the secretion of cytokines, including interferon- $\gamma$ (IFN- $\gamma$ ) and tumor necrosis factor- $\alpha$. Th2 cells downregulate antitumor immunity by secretion of cytokines, such as interleukin (IL)-4, IL-6 and IL-10 (24,25). A previous study determined that the Th1/Th2 balance may be disrupted in patients with HCC (26). Therefore, it is possible that aberrant expression of B7-H4 may be associated with the Th1/Th2 imbalance and contribute to HCC pathogenesis. The present study investigated the association between the expression levels of B7-H4 and HCC development.

\section{Materials and methods}

Cell culture. The H22 murine hepatoma cell line was obtained from the Department of Medicine \& Pharmacy Research Center of Binzhou Medical University (Binzhou, China). The HL-7702 normal human normal liver cell line and the Huh7 human hepatoma cell line were obtained from Cell Research Institute of the Chinese Academy of Sciences (Shanghai, China) and were cultured in Dulbecco's modified Eagle's medium (Hyclone; GE Healthcare Life Sciences, Logan, UT, USA) supplemented with $20 \%$ fetal bovine serum (Hyclone; GE Healthcare Life Sciences), 2 mM L-glutamine, 2 mM 4-(2-hydroxyethyl)-1-piperazineethanesulphonic acid, $100 \mathrm{mg} / \mathrm{ml}$ streptomycin and $100 \mathrm{U} / \mathrm{ml}$ penicillin in $5 \% \mathrm{CO}_{2}$ at $37^{\circ} \mathrm{C}$.

Establishment of tumor model. C57BL/6 mice were purchased from Weitong Lihua Experimental Animal Technical Co., Ltd. (Beijing, China) In total 30 mice were tumor-bearing mice and 18 were used as controls. Female and male mice (6-7 weeks old; 20-22 g) were housed at a ratio of 1:1 in a cage. All mice were housed six per cage at $20-24^{\circ} \mathrm{C}$ in a specific pathogen-free environment with a $12 \mathrm{~h}$ light/ $12 \mathrm{~h}$ dark cycle. Water and feed were sterilized by high pressure steam sterilization. All the animal experiments were performed following the ethical standards formulated by the Institutional Animal Experimental Ethics Committee of Binzhou Medical University.

$\mathrm{H} 22$ cells $\left(1 \times 10^{6}\right)$ were injected into the abdominal cavities of 6-7 week old C57BL/6 mice $(n=5)$. After 10 days, the mice were euthanized by cervical dislocation and a syringe was used to extract the ascites. The ascites were diluted to $5.0 \times 10^{5}$ cells $/ \mathrm{ml}$. Next, $0.2 \mathrm{ml} \mathrm{H} 22$ cells were subcutaneously injected into the right armpit of each mouse in order to establish tumors. After 24 h, 30 mice were randomly divided into three groups ( $\mathrm{n}=10$ per group). Tumors were established for 20,30 or 40 days in the three groups. Six mice were used as controls for each time point. The healthy control group received only saline injections. The mice were sacrificed via cervical dislocation after 20,30 and 40 days.

Reverse transcription-polymerase chain reaction (RT-PCR). Total mRNA was extracted from HL-7702 and Huh7 cells $\left(1 \times 10^{6}\right)$ using TRIzol (TaKaRa Biotechnology Co., Ltd., Dalian, China) according to the manufacturer's protocol. RT was performed using a PrimeScript RT-PCR kit (TaKaRa Biotechnology Co., Ltd.) according to the manufacturer's instructions. The following primers for $\mathrm{B} 7-\mathrm{H} 4$ and $\beta$-actin were obtained from Sangon Biotech Co., Ltd. (Shanghai, China): B7-H4, F 5'-AGGCTTCTCTGTGTGTCTCTTC-3', R 5'-CTTGCTCTTGTTTGCTCACTCC-3'; $\beta$-actin, F 5'-TTGTTACAGGAAGTCCCTTGCC-3', R 5'-ATGCTA TCACCTCCCCTGTGTG-3'. All reactions were performed using DNA polymerase from Transgen Biotech Co., Ltd. (Beijing, China) as follows: $95^{\circ} \mathrm{C}$ for $10 \mathrm{~min}, 95^{\circ} \mathrm{C}$ for $15 \mathrm{sec}$ and $60^{\circ} \mathrm{C}$ for $1 \mathrm{~min}$ for 35 cycles. PCR products were separated on $1 \%$ agarose gels and visualized by ethidium bromide staining.

Western blot analysis. Mouse tumor tissues were lysed using a lysis buffer (Beyotime Institute of Biotechnology, Haimen, China) and quantified using a bicinchoninic acid protein assay. Aliquots containing $20 \mu \mathrm{g}$ protein were dissolved in Laemmli buffer and incubated at $95^{\circ} \mathrm{C}$ for $10 \mathrm{~min}$. Proteins were separated on a $12 \%$ Tris-glycine SDS-PAGE (Beyotime Institute of Biotechnology), transferred onto polyvinylidene difluoride membranes (BD Pharmingen, San Diego, CA, USA) and incubated with 5\% non-fat dry milk in Tris-buffered saline/0.2\% Tween-20 (TBST) for $2 \mathrm{~h}$ at room temperature. The membranes were then incubated with anti-B7-H4 antibody (1:1,000 dilution; cat. no. ab108336; Abcam, Cambridge, UK) at $4^{\circ} \mathrm{C}$ overnight. Membranes were then washed three times with 1X TBST followed by incubation with horseradish peroxidase-conjugated goat anti-rabbit IgG secondary antibody (1:5,000; dilution; cat. no. ZB-2301; BIOSS, Beijing, China) for $1 \mathrm{~h}$ at room temperature. Membranes were washed five times in $1 \mathrm{X}$ TBST and proteins were visualized using an enhanced chemiluminescence kit (Roche Diagnostics, Basel, Switzerland). GAPDH was detected with mouse anti-GAPDH antibody (1:1,000 dilution; cat. no. AB-P-R 001; OriGene Technologies, Inc., Beijing, China) as an internal control.

Cytokine quantification. Fresh mouse tumor tissue supernatants, and mouse and human peripheral blood cells were lysed and centrifuged at $12,000 \mathrm{x} g$ at $4^{\circ} \mathrm{C}$ for $30 \mathrm{~min}$. Cytokine levels in supernatants were determined using enzyme-linked immunosorbent assay (ELISA) kits for IL-4 (human, cat. no. BP-E10142; mouse, cat. no. BP-E20011) IFN- $\gamma$ (human, BP-E10162; mouse, cat. no. BP-E11382), and soluble B7-H4 (sB7-H4; human, cat. no. BP-E11382; mouse, BP-E20905) from Shanghai Langdon Biotechnology Co., Ltd. (Shanghai, China) following the manufacturer's protocols. Standard curves served as internal controls for the sensitivity and range of each assay. Each sample was analyzed in triplicate.

Flow cytometry analysis. The expression level of B7-H4 in the HL-7702 and Huh7 cell lines was determined by flow cytometry. Cells were fixed and permeabilized using BD Perm Buffer III (BD Biosciences, Franklin Lakes, NJ, USA) for 20 mins at $4^{\circ} \mathrm{C}$. Samples were washed and incubated with purified mouse anti-human B7-H4 antibody (2 $\mu$ l per sample; cat. no. 562506; BD Biosciences) for 60 min and then with Alexa Fluor ${ }^{\circledR} 488$ donkey anti-mouse IgG H\&L (1:500; cat. no. ab150105; Abcam) in the dark for $30 \mathrm{~min}$ at $4^{\circ} \mathrm{C}$. Finally, the cells were washed and resuspended in Stain buffer (BD Biosciences) and 
A

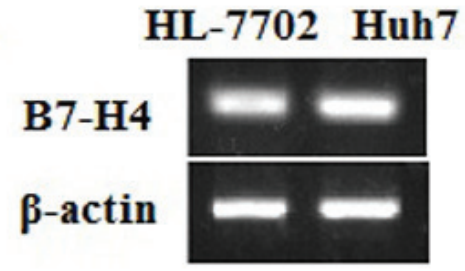

B

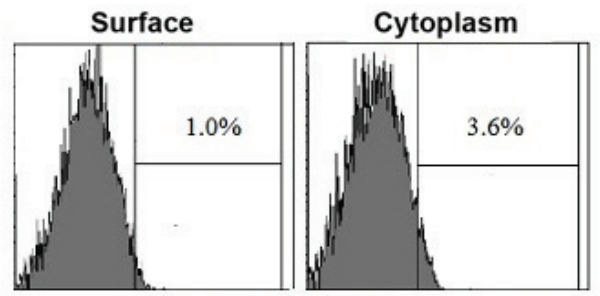

HL-7702 cell line

C

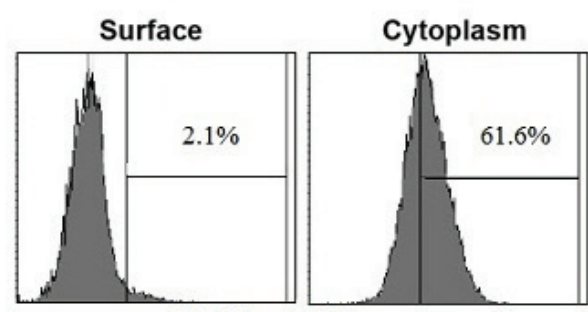

Huh7 cell line

D

$\mathbf{E}$
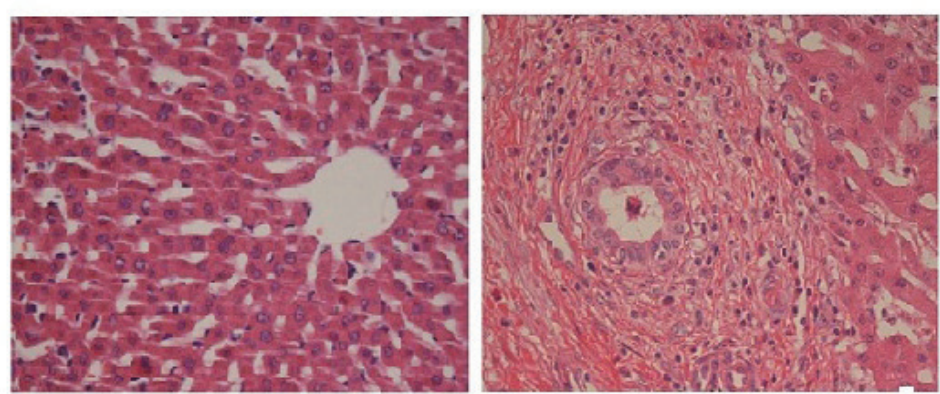

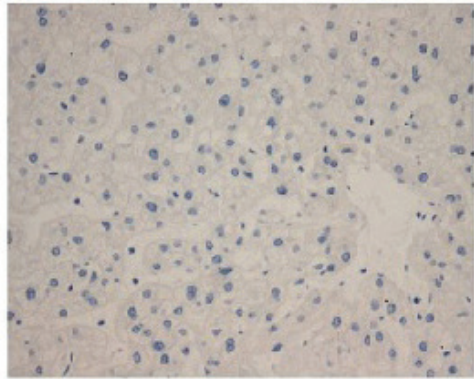

Normal liver tissue

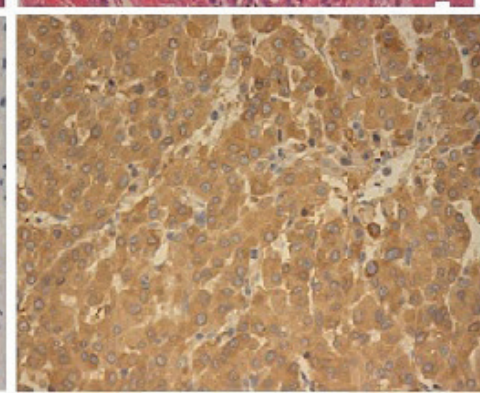

HCC tissue

Figure 1. (A) B7-H4 mRNA was detected in HL-7702 and Huh7 cells using reverse transcription-polymerase chain reaction. (B) B7-H4 expression was not observed in HL-7702 cells. (C) B7-H4 was detected on the cell surface and in the cytoplasm of Huh7 cells. The expression levels in cytoplasm were higher compared with the cell surface. (D) H\&E staining of normal human liver tissue and HCC tissues. (E) Immunohistochemistry was used to evaluate B7-H4 expression in normal liver and HCC tissues. B7-H4 expression was not detected in normal liver tissues. Intense B7-H4 staining was observed in HCC tissue. Magnification, x400. HCC, hepatocellular carcinoma; H\&E, hematoxylin and eosin.

analyzed on a BD FACSCalibur. The data were analyzed with BD FACSDiva 7.0 software (BD Biosciences).

Patients. Patients with HCC were recruited from Yu Huang Ding Hospital (Yantai, China) from August 2013 to January 2015. The protocol for the present study was approved by University of Binzhou Medical College Ethics Committee and informed consent was obtained from all patients. The present study included patients with HCC $(n=60)$ and healthy controls $(n=20)$. HCC was diagnosed on the basis of biochemistry and image findings, including sonography, computerized tomography scans, or magnetic resonance imaging scans. 
Table I. Clinical and pathological features of patients with hepatocellular carcinoma.

\begin{tabular}{|c|c|c|c|c|}
\hline \multirow[b]{2}{*}{ Clinical characteristic } & \multirow[b]{2}{*}{ Number } & \multicolumn{2}{|c|}{ B7-H4 expression } & \multirow[b]{2}{*}{ P-value ${ }^{a}$} \\
\hline & & Negative & Positive & \\
\hline Gender & & & & 0.895 \\
\hline Male & 35 & 19 & 16 & \\
\hline Female & 25 & 14 & 11 & \\
\hline Age & & & & 0.979 \\
\hline$<60$ & 29 & 16 & 13 & \\
\hline$\geq 60$ & 31 & 17 & 14 & \\
\hline TNM stages & & & & 0.004 \\
\hline $\mathrm{I}+\mathrm{II}$ & 28 & 21 & 7 & \\
\hline III+IV & 32 & 12 & 20 & \\
\hline Differentiation degree & & & & 0.007 \\
\hline Well/moderate & 27 & 20 & 7 & \\
\hline Poor & 33 & 13 & 20 & \\
\hline Lymph node metastasis & & & & 0.002 \\
\hline Yes & 25 & 8 & 17 & \\
\hline No & 35 & 25 & 10 & \\
\hline Size of tumor $(\mathrm{cm})$ & & & & 0.979 \\
\hline$<4$ & 29 & 16 & 13 & \\
\hline$\geq 4$ & 31 & 17 & 14 & \\
\hline Intravascular cancer embolus & & & & 0.802 \\
\hline Yes & 19 & 10 & 9 & \\
\hline No & 41 & 23 & 18 & \\
\hline \multicolumn{5}{|c|}{$\begin{array}{l}\text { Hepatocellular carcinoma-associated } \\
\text { tumor antigens }\end{array}$} \\
\hline CEA $(0.5 \mathrm{ng} / \mathrm{ml})$ & & & & 0.714 \\
\hline$<5$ & 26 & 15 & 11 & \\
\hline$\geq 5$ & 34 & 18 & 16 & \\
\hline CA19-9 (0-27 U/ml) & & & & 0.895 \\
\hline$<27$ & 25 & 14 & 11 & \\
\hline$\geq 27$ & 35 & 19 & 16 & \\
\hline $\operatorname{AFP}(0-27$ ng/ml $)$ & & & & 0.176 \\
\hline$<400$ & 17 & 7 & 10 & \\
\hline$\geq 400$ & 43 & 26 & 17 & \\
\hline
\end{tabular}

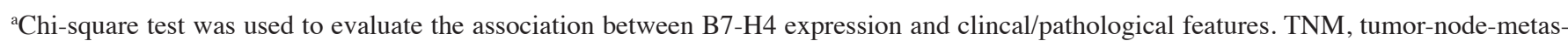
tasis; CEA, carcinoembryonic antigen; CA19-9, carbohydrate antigen 19-9; AFP, $\alpha$ fetoprotein.

All samples were examined histologically and diagnosis was conducted according to the American Association for the Study of Liver Diseases guidelines (9).

Immunohistochemical staining. B7-H4 expression was analyzed in human normal liver tissues, HCC tissues and mouse tumor tissues using immunohistochemistry staining. The B7-H4 primary antibody used was obtained from Abcam, the secondary biotin-labeled goat-rabbit antibody and diaminobenzidine tetrahydrochloride solution were provided by Boster Biological Technology, Ltd. (Wuhan, China). All samples were fixed in formalin solution and embedded in paraffin. Sections
(5 $\mu \mathrm{m}$-thick) were dewaxed in xylene, dehydrated in ethanol, and incubated in $3 \% \mathrm{H}_{2} \mathrm{O}_{2}$ for $20 \mathrm{~min}$. Following incubation in $5 \%$ normal bovine serum (Boster Biological Technology, Ltd.) for $20 \mathrm{~min}$, slides were incubated with the primary antibody at $4^{\circ} \mathrm{C}$ overnight, and then with the secondary antibody at $37^{\circ} \mathrm{C}$ for $60 \mathrm{~min}$. Negative controls were established by replacing the primary antibody with normal mouse IgG (BD Pharmingen). Slides were visualized using light microscopy.

Evaluation of immunostaining. Immunostaining was independently examined by two clinical pathologists. Five high-power fields were randomly selected per sample. 
Table II. Serum level of sB7-H4, IFN- $\gamma$ and IL-4 are changed in different clinical stages.

\begin{tabular}{|c|c|c|c|c|c|c|c|}
\hline \multirow[b]{2}{*}{ Group } & \multirow[b]{2}{*}{$\mathrm{n}$} & \multicolumn{2}{|c|}{ sB7-H4 (ng/ml) } & \multicolumn{2}{|c|}{ IL-4 (ng/ml) } & \multicolumn{2}{|c|}{$\mathrm{IFN}-\gamma(\mathrm{pg} / \mathrm{m})$} \\
\hline & & Mean \pm SD & P-value ${ }^{a}$ & Mean \pm SD & P-value ${ }^{a}$ & Mean \pm SD & P-value ${ }^{a}$ \\
\hline I-II stages & 28 & $41.94 \pm 4.68$ & $<0.001$ & $600.87 \pm 13.55$ & $<0.001$ & $247.08 \pm 6.48$ & $<0.001$ \\
\hline III-IV stages & 32 & $74.93 \pm 16.28$ & & $796.41 \pm 42.10$ & $<0.001$ & $163.46 \pm 14.94$ & \\
\hline
\end{tabular}

aI-II stages vs. III-IV stages. sB7-H4, soluble B7-H4; IL-4, interleukin-4; IFN- $\gamma$, interferon- $\gamma$; SD, standard deviation.

A

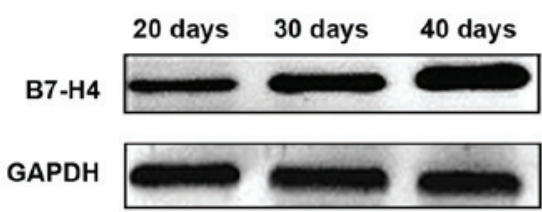

C

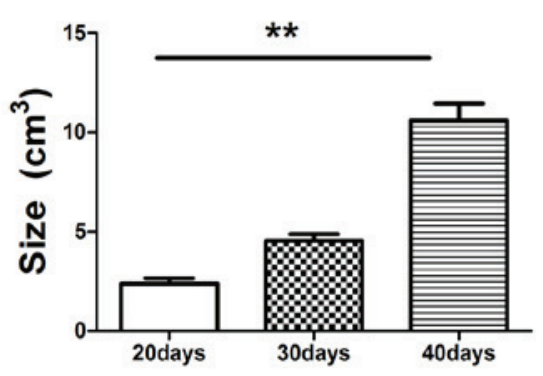

B

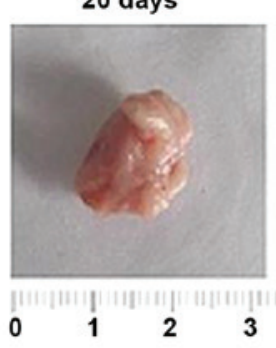

30 days

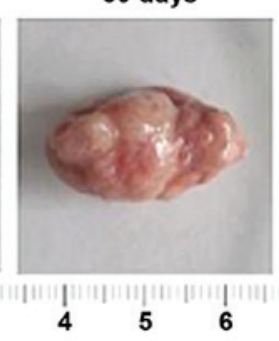

40 days

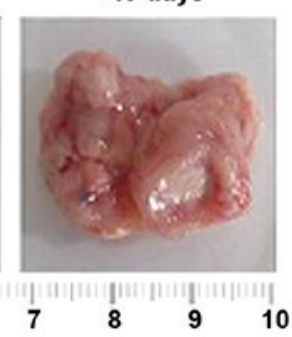

D

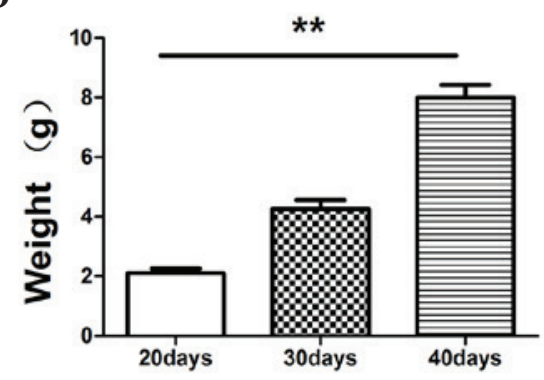

E

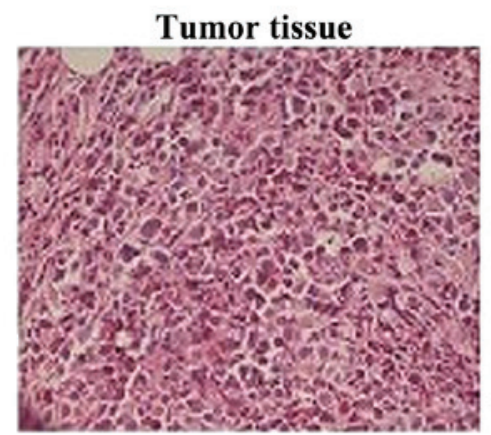

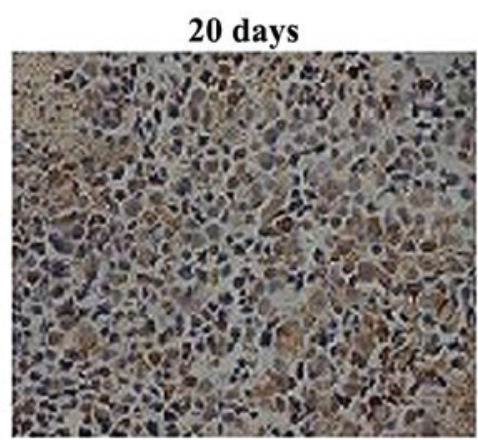

30 days

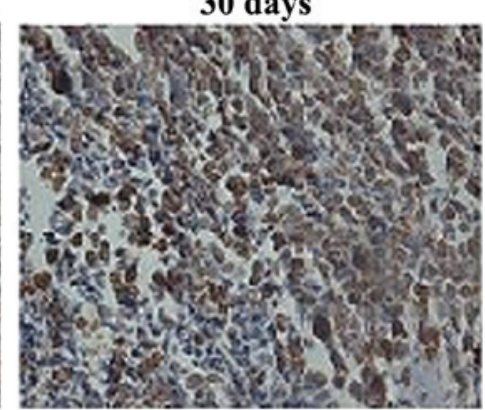

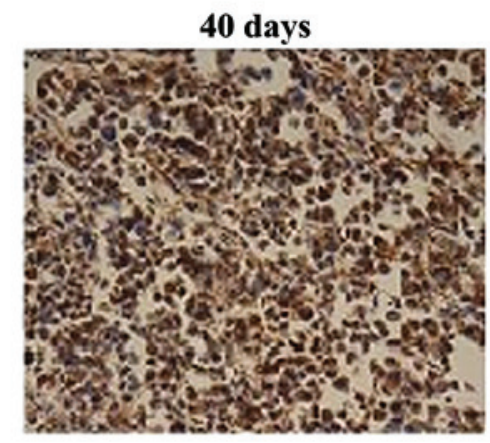

Control

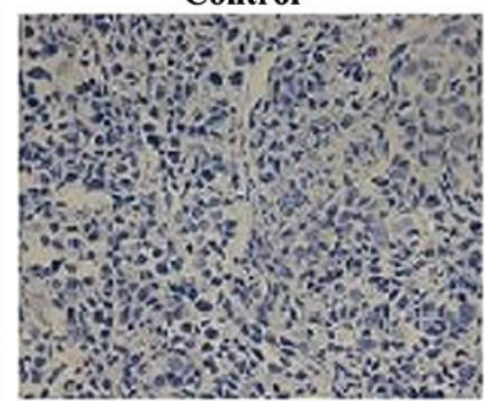

Figure 2. B7-H4 was detected in tumor tissues using western blotting at 20, 30, and 40 days following the tumor cell injection. (A) B7-H4 increased with time. (B) Tumor size also increased with time as presented in the photographs of representative tumors. (C) Quantification of tumor size increase with time. ${ }^{* *} \mathrm{P}<0.001$ (D) Tumor weight increased with time. ${ }^{* *} \mathrm{P}<0.001$. (E) B7-H4 immunohistochemistry and H\&E staining in mouse tumor model. Representative H\&E staining of mouse tumor tissue. Immunohistochemical staining was performed on normal tissue and tumor tissue at 20, 30, and 40 days to determine B7-H4 expression levels. Magnification, $\mathrm{x} 400 . \mathrm{H} \& \mathrm{E}$, hematoxylin and eosin. 
A

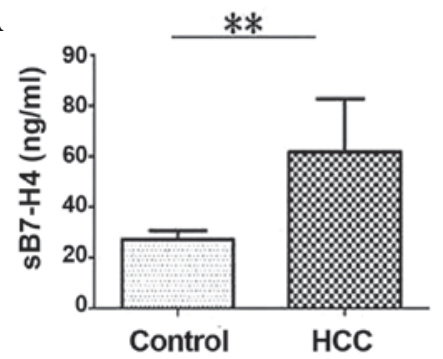

D

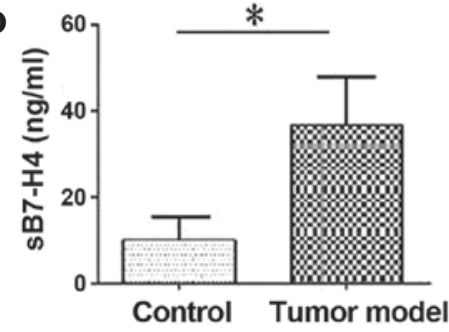

B

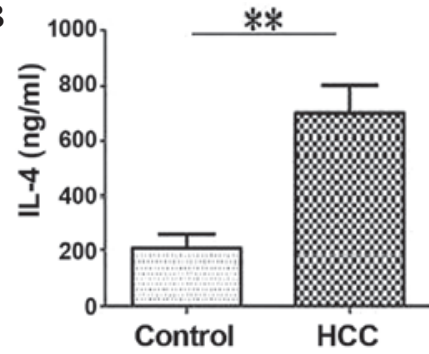

E

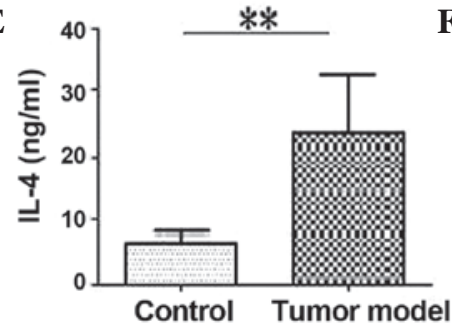

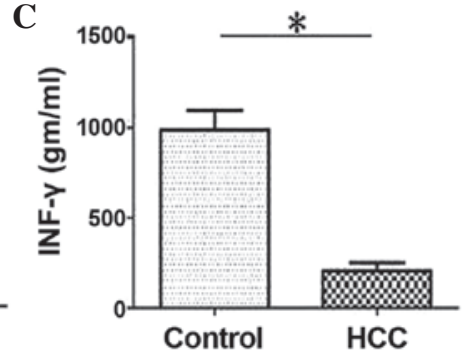

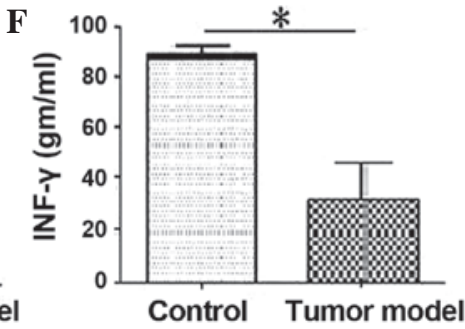

Figure 3. Analysis of sB7-H4, IFN- $\gamma$ and IL-4 in HCC patients, tumorigenic mice and healthy controls. (A) sB7-H4 $\left({ }^{* *} \mathrm{P}<0.001\right)$ and (B) IL-4 levels $\left({ }^{* *} \mathrm{P}<0.001\right)$ were increased and (C) IFN- $\gamma$ levels (" $\mathrm{P}=0.017$ ) were reduced in HCC patients compared with healthy controls. (D) sB7-H4 ("P=0.018) and (E) IL-4 levels $\left({ }^{* * *} \mathrm{P}=0.004\right)$ were increased and $(\mathrm{F}) \mathrm{IFN}-\gamma$ levels $\left({ }^{*} \mathrm{P}=0.012\right)$ were reduced in serum from tumorigenic mice compared with those in healthy controls. sB7-H4, soluble B7-H4; IL-4, interleukin-4; IFN- $\gamma$, interferon- $\gamma$; HCC, hepatocellular carcinoma.

Staining intensity of positive tumor cells was assessed. The extent of the staining was categorized into five semiquantitative classes based on the percentages of membrane-positive tumor cells: i) 0 ( $<5 \%$ positive tumor cells); ii) 1 (6-25\% positive tumor cells); iii) 2 (26-50\% positive tumor cells); iii) 3 (51-75\% positive tumor cells); and iv) 4 ( $>75 \%$ positive tumor cells). The intensity of staining was determined semiquantitatively on a scale of 0 to 3 as follows: i) 0 (negative); ii) 1 (weakly positive), iii) 2 (moderately positive); and iv) 3 (strongly positive). Multiplication of the intensity and the percentage scores was used to obtain the final staining scores, 0 (negative), + (1-2), ++ (3-4), and +++ (5-7).

Statistical analysis. For all statistical analyses, data were processed with SPSS version 17.0 statistical software (SPSS, Inc., Chicago, IL, USA). Correlation of B7-H4 protein expression with Clinical and pathological features of patients with hepatocellular carcinoma was evaluated with the Chi-square test. The correlations of the expression levels of sB7-H4, IL-4 and IFN- $\gamma$ were analyzed by Spearman correlation coefficients. Two independent sample t-test was used to analyze the significance of B7-H4 expression scores between the HCC group and the control group. For comparison of three or more groups, one-way analysis of variance was performed. Data are presented as the mean \pm standard deviation. $\mathrm{P}<0.05$ was considered to indicate a statistically significant difference

\section{Results}

B7-H4 expression in HL-7702 and Huh7 cells. The expression of B7-H4 in the cell lines was analyzed using RT-PCR and flow cytometry. B7-H4 transcripts were expressed in both HL-7702 and Huh7 cell lines as presented in Fig. 1A. The B7-H4 protein was not detected in HL-7702 cells (Fig. 1B). B7-H4 was detected in Huh7 cells, with the expression levels higher in the cytoplasm compared with the cell surface (Fig. 1C).
Expression of B7-H4 in normal liver and HCC tissues. In order to determine whether B7-H4 was differentially expressed in human normal liver and HCC tissues, immunohistochemical analysis was performed. $\mathrm{B} 7-\mathrm{H} 4$ protein was not expressed in human normal liver tissues; however, it was expressed in HCC tissues. B7-H4 was observed in the cytoplasm and membrane of HCC cells; however, it was not detected in the nucleus (Fig. 1D and E).

B7-H4 expression and clinicopathological features. Immunohistochemical analysis was used to examine B7-H4 expression in patients with HCC (Table I). The patients included 35 males and 25 females with an age range of 47 to 77 years (median, 61.9 years). A total of 27 patients had well or moderately-well differentiated cancer cells and in 33 patients cancer cells were poorly differentiated, 25 patients had lymph node metastasis. Patients were staged from I to IV based on American Joint Committee on Cancer standards. There were 28 patients in stages I and II and 32 patients in stages III and IV. Patient characteristics and clinicopathological features are summarized in Table I.

B7-H4 expression in tumor tissues was significantly positively correlated with TNM stage, differentiation degree and lymph node metastasis $(\mathrm{P}<0.05$; Table I). No association between B7-H4 expression and the remaining factors, including gender, age, tumor size or hepatoma carcinoma-associated tumor antigens was identified (Table I). Therefore, this indicated that the expression of $\mathrm{B} 7-\mathrm{H} 4$ is associated with aggressive $\mathrm{HCC}$.

B7-H4 expression as a function of time in mouse tumor model. The mouse liver tumor model was established by subcutaneous injection of $\mathrm{H} 22$ cells into mice. B7-H4 in tumor tissues was detected by western blotting (Fig. 2A). The sizes and weights of tumors were evaluated at 20,30, and 40 days and were found to significantly increase with time $(\mathrm{P}<0.01$; Fig. $2 \mathrm{~B}-\mathrm{D})$. The expression of B7-H4 in tumor tissues was confirmed by 

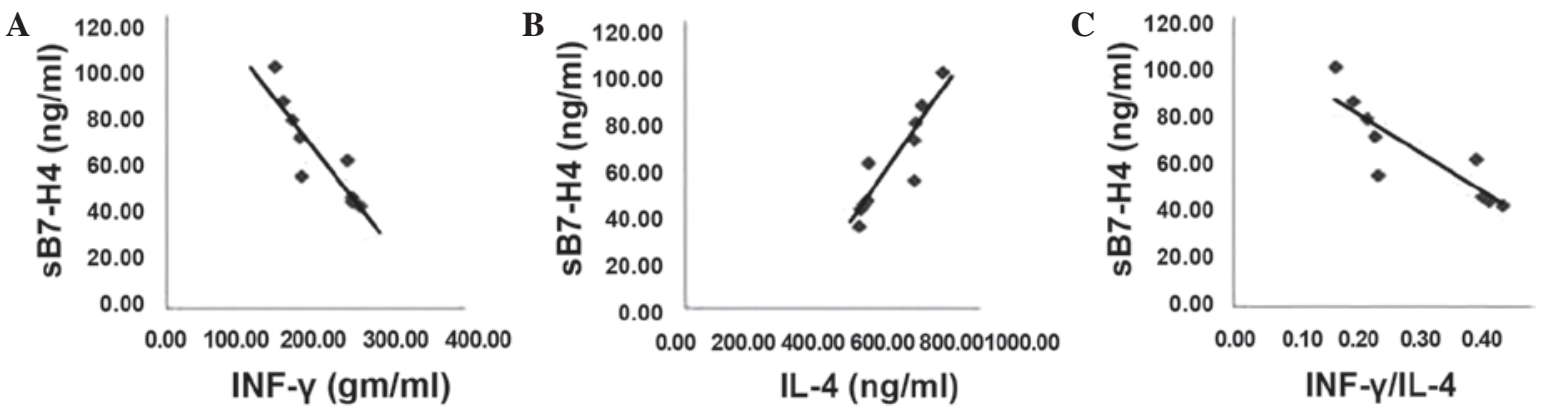

Figure 4. Correlations observed between levels of sB7-H4, IFN- $\gamma$, and IL-4 and the ratio of IFN- $\gamma / \mathrm{IL}-4$ in serum collected from patients with HCC. Levels of sB7-H4 vs. (A) IFN- $\gamma$ levels (B) IL-4 levels and (C) the ratio of IFN- $\gamma /$ IL-4 in patients with HCC. sB7-H4, soluble B7-H4; IL-4, interleukin-4; IFN- $\gamma$, interferon- $\gamma$; HCC, hepatocellular carcinoma.

A

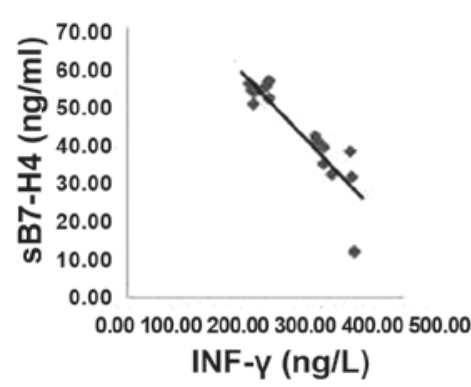

D

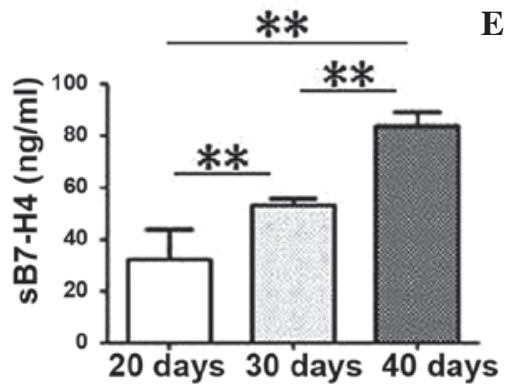

B

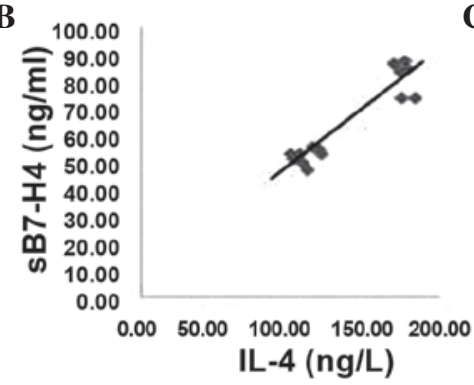

C

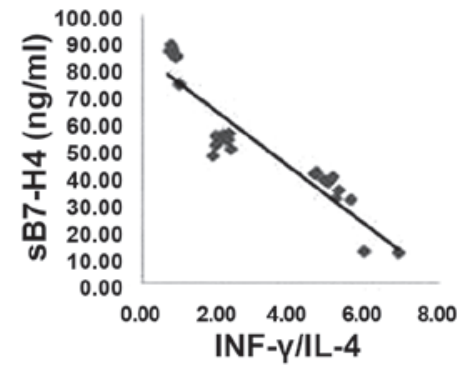

$\mathbf{F}$

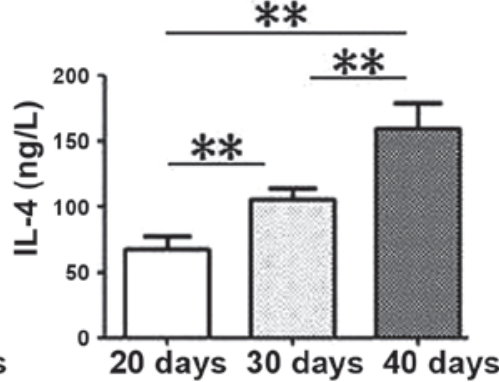

20 days 30 days 40 days

G
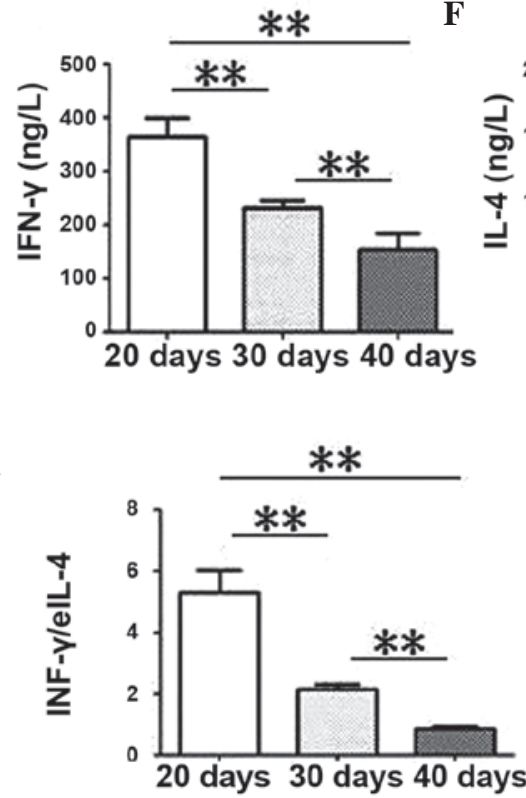

Figure 5. Correlations observed between the levels of sB7-H4, IFN- $\gamma$, and IL-4 and the ratio of IFN- $\gamma / \mathrm{IL}-4$ in mouse tumor samples. (A) sB7-H4 levels correlated negatively with IFN- $\gamma$ levels in mouse tumor tissues. (B) sB7-H4 levels correlated positively with IL-4 levels in mouse tumor tissue. (C) sB7-H4 levels correlated negatively with the ratio of IFN- $\gamma / \mathrm{IL}-4$ in tumor tissue. Expression levels of (D) sB7-H4, (E) IFN- $\gamma$, (F) IL-4 and (G) ratio of IFN- $\gamma / \mathrm{IL}-4$ at different times following the injection with tumor cells. ${ }^{* *} \mathrm{P}<0.01$. sB7-H4, soluble B7-H4; IL-4, interleukin-4; IFN- $\gamma$, interferon- $\gamma$.

immunohistochemistry (Fig. 2E). At 20 days, B7-H4 levels were $3.28 \pm 0.47$; at 30 days, levels were $5.36 \pm 0.38$ and at 40 days, levels were $6.18 \pm 0.32$. These results indicated that the expression levels of B7-H4 increased as tumors increased in size and weight.

Analysis of sB7-H4, IFN- $\gamma$ and IL-4 in HCC patients, tumorigenic mice and healthy controls. Cytokine levels in the blood serum were analyzed using an ELISA. The results revealed that sB7-H4 and IL-4 levels in HCC patients were significantly higher compared with healthy controls ( $\mathrm{P}<0.001$; Fig. $3 \mathrm{~A}$ and $\mathrm{B})$. IFN- $\gamma$ levels were significantly reduced in patients with HCC compared with the healthy control group $(\mathrm{P}=0.017$; Fig. $3 \mathrm{C})$. The results revealed that sB7-H4 (P=0.018; Fig. 3D) and IL-4 $(\mathrm{P}=0.004$; Fig. 3E) levels in the serum of tumor-carrying mice were significantly higher compared with healthy controls. 

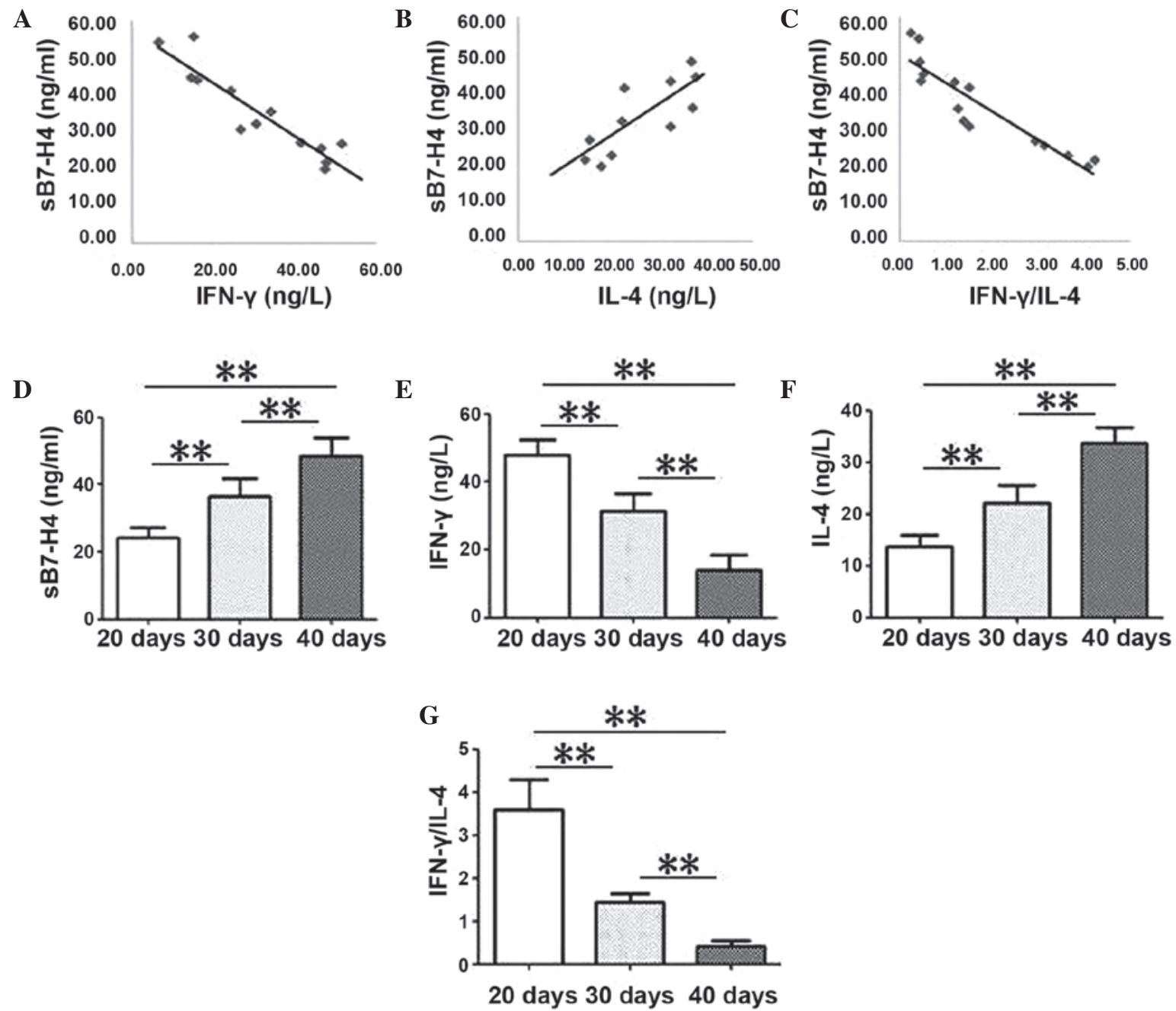

Figure 6. Correlations observed between the levels of sB7-H4, IFN- $\gamma$, and IL-4 and the ratio of IFN- $\gamma /$ IL-4 in mouse serum samples. (A) sB7-H4 levels correlated negatively with IFN- $\gamma$ levels in tumorigenic mouse serum samples. (B) sB7-H4 levels correlated positively with IL-4 levels in tumorigenic mouse serum samples. (C) sB7-H4 levels correlated negatively with the ratio of IFN- $\gamma /$ IL-4 in mouse serum samples. Levels of (D) sB7-H4, (E) IFN- $\gamma$, (F) IL-4, and (G) ratio of IFN- $\gamma /$ IL-4 as a function of time in tumorigenic mouse serum samples. ** $\mathrm{P}<0.01$. sB7-H4, soluble B7-H4; IL-4, interleukin-4; IFN- $\gamma$, interferon- $\gamma$.

IFN- $\gamma$ levels were significantly reduced in patients with $\mathrm{HCC}$ compared with the healthy control group $(\mathrm{P}=0.012$; Fig. $3 \mathrm{~F})$.

A Spearman's rank correlation analysis was used to identify the correlations between sB7-H4 levels and IFN- $\gamma$, IL- 4 and the ratio between IFN- $\gamma /$ IL- 4 levels in blood serum samples of patients with HCC (Fig. 4). sB7-H4 levels correlated negatively with IFN- $\gamma$ and with the ratio of IFN- $\gamma / \mathrm{IL}-4$ $(\mathrm{R}=-0.888 ; \mathrm{P}=0.001$ and $\mathrm{R}=-0.864 ; \mathrm{P}=0.003$, Fig. $4 \mathrm{~A}$ and $\mathrm{C})$. Conversely, sB7-H4 levels were positively correlated with the levels of IL-4 in the blood serum samples of patients with HCC ( $\mathrm{R}=0.903, \mathrm{P}<0.001$; Fig. 4C).

Spearman's rank was also used to determine correlations between the cytokine levels in tumor samples (Fig. 5) and serum samples (Fig. 6) from the mouse tumor model. In tumor tissue samples from mice, sB7-H4 levels were negatively correlated with IFN- $\gamma$ levels and with the ratio of IFN- $\gamma / \mathrm{IL}-4(\mathrm{R}=-0.919$, $\mathrm{P}<0.001$; and $\mathrm{R}=-0.925, \mathrm{P}<0.001$, respectively; Fig. 5A and $\mathrm{B}$ ). Negative correlations were also observed in serum samples obtained from the mice $(\mathrm{R}=-0.942 ; \mathrm{P}<0.001$ and $\mathrm{R}=-0.923$; $\mathrm{P}<0.001$; Fig. 6A and C). sB7-H4 levels were positively correlated with IL-4 levels from mouse tumor tissue $(\mathrm{R}=0.951$; $\mathrm{P}<0.001$; Fig. 5A) and serum samples $(\mathrm{R}=0.917$; $\mathrm{P}<0.001$; Fig. 6B).
IFN- $\gamma$ levels and the ratio of IFN- $\gamma /$ IL-4 significantly decreased over time in tumor tissue $(\mathrm{P}<0.001$; Fig. $5 \mathrm{E}$ and $\mathrm{G})$ and serum samples $(\mathrm{P}<0.001$; Fig. $6 \mathrm{E}$ and $\mathrm{G})$. Conversely, sB7-H4 and IL-4 levels in mouse tumor tissues $(\mathrm{P}<0.001$; Fig. 5D and F) and serum samples $(\mathrm{P}<0.001$; Fig. $6 \mathrm{D}$ and $\mathrm{F})$ significantly increased with time.

sB7-H4, IFN- $\gamma$ and IL-4 levels are altered with the clinical stage of HCC. In order to determine whether the expression levels of sB7-H4, IFN- $\gamma$, and IL-4 are associated with the patient's HCC clinical stage, ELISA analysis was used (Table II). sB7-H4 and IL-4 levels were increased in stage III and IV tumors compared with stage I and II tumors $(\mathrm{P}<0.001$; Table II), whereas IFN- $\gamma$ levels were increased in stage III and IV tumors compared with stage I and II tumors $(\mathrm{P}<0.001$; Table II). Therefore, this indicated that sB7-H4 may be a potential marker to predict tumor progression in patients with HCC.

\section{Discussion}

HCC is a common malignant tumor with high morbidity and mortality, particularly among patients in China (27). Previous 
studies have determined that B7-H4 expression may be important for tumorigenesis (15-20).

B7-H4 is a member of the B7 family of costimulatory ligands. It has a negative regulatory function in $\mathrm{T}$ cell-mediated immunity as it has been identified to inhibit $\mathrm{T}$ cell activation, proliferation and cytokine production (10-12). Notably, despite widespread $\mathrm{B} 7-\mathrm{H} 4 \mathrm{mRNA}$ expression, the expression levels of the B7-H4 protein have been observed to be restricted in normal tissues (28). Previous studies have demonstrated that the B7-H4 molecule is highly expressed in various different types of human cancers, including pancreatic (16) and gastric cancer $(17,18)$. The present study revealed that the $\mathrm{B} 7-\mathrm{H} 4$ protein was not expressed by the HL-7702 normal human liver cell line; however, it was expressed by the Huh7 human hepatoma cells. Immunohistochemical staining revealed that B7-H4 was expressed in HCC tissues and not in normal liver tissues. In addition, in a mouse tumor model was established using the $\mathrm{H} 22$ cell line. B7-H4 protein expression was observed in the tumor tissues obtained from the mice. These results indicate an association between $\mathrm{B} 7-\mathrm{H} 4$ expression and HCC.

Previous studies determined that B7-H4 had no prognostic value for ovarian cancer (29) or breast cancer (30). However, the expression levels of $\mathrm{B} 7-\mathrm{H} 4$ in renal cell carcinoma (RCC) (31), gastric cancer $(17,18)$ and colorectal carcinoma (19) have been identified to be correlated with adverse clinicopathological features, such as advanced lymph node metastasis, tumor grade and TNM stage. Prostate carcinoma and patients with RCC, that have B7-H4-positive tumors have also been identified to be at a high risk of recurrence and had increased mortality (32). The present study determined that higher B7-H4 expression levels were observed in samples from patients with poor differentiation and lymph node metastasis and at later stages of progression. In the mouse model, the expression levels of B7-H4 increased with time. These results suggested that B7-H4 is important for the progression of $\mathrm{HCC}$, B7-H4 may also be used as a molecular marker of HCC and as a novel target for HCC therapy.

A previous study determined that serum B7-H4 levels were significantly increased in patients with gastric cancer compared with healthy volunteers, additionally high sB7-H4 levels were significantly correlated with tumor size, lymph node metastasis and TNM stage in patients with gastric cancer (33). Simon et al (34) determined that B7-H4 expression was elevated in serum samples from ovarian cancer patients when compared with healthy controls or women with benign gynecologic diseases, including endometriosis, enlarged ovaries/edema and polycystic ovaries. Zhang et al (35) revealed that sB7-H4 levels in patients with $\mathrm{HCC}$ were significantly higher compared with those in normal controls and that sB7-H4 levels were closely associated with tumor size, tumor invasion, tumor differentiation and TNM stage (35). However, they were not associated with other characteristics, including age, gender and alanine aminotransferase levels (35). In the present study, elevated expression levels of sB7-H4 were observed in blood samples from patients with HCC compared with healthy controls, which was consistent with previous studies. Additionally, the current study determined that the levels of IL-4 were higher and those of IFN- $\gamma$ lower in serum samples from patients with HCC compared with serum samples from healthy controls. sB7-H4 levels were negatively correlated with IFN- $\gamma$ levels and with the ratio of IFN- $\gamma /$ IL-4. However, sB7-H4 levels were positively correlated with IL-4 levels in serum samples obtained from patients with HCC. Similar results were observed in serum and tumor tissues samples obtained from the mouse model. This suggests that the expression levels of sB7-H4 may be due to an imbalance of Th1 and Th2 cells, that facilitates the development of HCC. In addition, the present study also determined that sB7-H4 and IL-4 levels were positively correlated with the TNM stage in HCC patients, whereas IFN- $\gamma$ levels were negatively correlated. Therefore, sB7-H4 may be used as a potential serum biomarker to facilitate diagnosis of HCC and may be predictive of tumor progression in patients with HCC.

The present study revealed a possible role for B7-H4 in the development of HCC. Aberrant expression of B7-H4 has been identified to correlate with the TNM stage, differentiation degree and lymph node metastasis in patients with HCC. The present study provided insight into the underlying mechanism that contributed to the progression of HCC and suggested that B7-H4 may be a promising target for immunotherapy.

\section{Acknowledgements}

The present study was supported by funds from the Nature Science Foundation of Shandong Province (grant no. ZR2013HM050) and the Foundation Project in Shandong Province Department of Education (J02K12).

\section{References}

1. Calle EE, Rodriguez C, Walker-Thurmond $\mathrm{K}$ and Thun MJ: Overweight, obesity, and mortality from cancer in a prospectively studied cohort of US adults. N Engl J Med 348: 1625-1638, 2003.

2. Parkin DM: Global cancer statistics in the year 2000. Lancet Oncol 2: 533-543, 2001.

3. Schütte K, Bornschein J and Malfertheiner P: Hepatocellular carcinoma-epidemiological trends and risk factors. Dig Dis 27: 80-92, 2009.

4. Hawkins MA and Dawson LA: Radiation therapy for hepatocellular carcinoma: From palliation to cure. Cancer 106: 1653-1663, 2006.

5. Yang JD and Roberts LR: Hepatocellular carcinoma: A global view. Nat Rev Gastroenterol Hepatol 7: 448-458, 2010.

6. Parkin DM, Bray F, Ferlay J and Pisani P: Global cancer statistics 2002. CA Cancer J Clin 55: 74-108, 2005.

7. El-Serag HB, Marrero JA, Rudolph L and Reddy KR: Diagnosis and treatment of hepatocellular carcinoma. Gastroenterology 134: 1752-1763, 2008

8. Kudo M, Izumi N, Kokudo N, Matsui O, Sakamoto M, Nakashima O, Kojiro M and Makuuchi M; HCC Expert Panel of Japan Society of Hepatology: Management of hepatocellular carcinoma in Japan: Consensus-based clinical practice guidelines proposed by the Japan Society of Hepatology (JSH) 2010 updated version. Dig Dis 29: 339-364, 2011.

9. Bruix J and Sherman M; Practice Guidelines Committee, American Association for the Study of Liver Diseases: Management of hepatocellular carcinoma. Hepatology 42: 1208-1236, 2005.

10. Chen L: Co-inhibitory molecules of the B7-CD28 family in the control of T-cell immunity. Nat Rev Immunol 4: 336-347, 2004.

11. Sica GL, Choi IH, Zhu G, Tamada K, Wang SD, Tamura H, Chapoval AI, Flies DB, Bajorath J and Chen L: B7-H4, a molecule of the B7 family, negatively regulates $\mathrm{T}$ cell immunity. Immunity 18: 849-861, 2003. 
12. Zang X, Loke P, Kim J, Murphy K, Waitz R and Allison JP B7x: A widely expressed B7 family member that inhibits $T$ cell activation. Proc Natl Acad Sci USA 100: 10388-10392, 2003.

13. Prasad DV, Richards S, Mai XM and Dong C: B7S1, a novel $\mathrm{B} 7$ family member that negatively regulates $\mathrm{T}$ cell activation. Immunity 18: 863-873, 2003.

14. Flies DB and Chen L: The new B7s: Playing a pivotal role in tumor immunity. J Immunother 30: 251-260, 2007.

15. Chen LJ, Sun J, Wu HY, Zhou SM, Tan Y, Tan M, Shan BE, Lu BF and Zhang XG: B7-H4 expression associates with cancer progression and predicts patient's survival in human esophageal squamous cell carcinoma. Cancer Immunol Immunother 60: 1047-1055, 2011.

16. Awadallah NS, Shroyer KR, Langer DA, Torkko KC, Chen YK, Bentz JS, Papkoff J, Liu W, Nash SR and Shah RJ: Detection of B7-H4 and p53 in pancreatic cancer: Potential role as a cytological diagnostic adjunct. Pancreas 36: 200-206, 2008.

17. Arigami T, Uenosono Y, Ishigami S, Hagihara T, Haraguchi N and Natsugoe S: Clinical significance of the B7-H4 coregulatory molecule as a novel prognostic marker in gastric cancer. World J Surg 35: 2051-2057, 2011.

18. Geng Y, Wang H, Lu C, Li Q, Xu B, Jiang J and Wu C: Expression of costimulatory molecules B7-H1, B7-H4 and Foxp3+ Tregs in gastric cancer and its clinical significance. Int J Clin Oncol 20 : 273-281, 2015

19. Zhao LW, Li C, Zhang RL, Xue HG, Zhang FX, Zhang F and Gai XD: B7-H1 and B7-H4 expression in colorectal carcinoma: Correlation with tumor FOXP3(+) regulatory T-cell infiltration Acta Histochem 116: 1163-1168, 2014.

20. Chen C, Qu QX, Shen Y, Mu CY, Zhu YB, Zhang XG and Huang JA: Induced expression of B7-H4 on the surface of lung cancer cell by the tumor-associated macrophages: A potential mechanism of immune escape. Cancer Lett 317: 99-105, 2011.

21. Jeon H, Vigdorovich V, Garrett-Thomson SC, Janakiram M, Ramagopal UA, Abadi YM, Lee JS, Scandiuzzi L, Ohaegbulam KC, Chinai JM, et al: Structure and cancer immunotherapy of the B7 family member B7x.Cell Rep 9: 1089-1098, 2014.

22. Kryczek I, Zou L, Rodriguez P, Zhu G, Wei S, Mottram P, Brumlik M, Cheng P, Curiel T, Myers L, et al: B7-H4 expression identifies a novel suppressive macrophage population in human ovarian carcinoma. J Exp Med 203: 871-881, 2006.

23. Zhu J and Paul WE: CD4 T cells: Fates, functions, and faults. Blood 112: 1557-1569, 2008.

24. Nieters A, Yuan JM, Sun CL, Zhang ZQ, Stoehlmacher J, Govindarajan S and Yu MC: Effect of cytokine genotypes on the hepatitis B virus-hepatocellular carcinoma association. Cancer 103: 740-748, 2005.
25. Ognjanovic S, Yuan JM, Chaptman AK, Fan Y and Yu MC: Genetic polymorphisms in the cytokine genes and risk of hepatocellular carcinoma in low-risk non-Asians of USA. Carcinogenesis 30: 758-762, 2009.

26. Zhou D, Gu FM, Gao Q, Li QL, Zhou J and Miao CH: Effects of anesthetic methods on preserving anti-tumor T-helper polarization following hepatectomy. World J Gastroenterol 18: 3089-3098, 2012.

27. Li W, Huang X, Tong H, Wang Y, Zhang T, Wang W, Dai L, $\mathrm{Li} \mathrm{T}$, Lin S and Wu H: Comparison of the regulation of $\beta$-catenin signaling by type I, type II and type III interferons in hepatocellular carcinoma cells. PLoS One 7: e47040, 2012.

28. Choi IH, Zhu G, Sica GL, Strome SE, Cheville JC, Lau JS, Zhu Y, Flies DB, Tamada $\mathrm{K}$ and Chen L: Genomic organization and expression analysis of B7-H4, an immune inhibitory molecule of the B7 family. J Immunol 171: 4650-4654, 2003.

29. Tringler B, Liu W, Corral L, Torkko KC, Enomoto T, Davidson S, Lucia MS, Heinz DE, Papkoff J and Shroyer KR: B7-H4 overexpression in ovarian tumors. Gynecol Oncol 100: 44-52, 2006.

30. Tringler B, Zhuo S, Pilkington G, Torkko KC, Singh M, Lucia MS, Heinz DE, Papkoff J and Shroyer KR: B7-H4 is highly expressed in ductal and lobular breast cancer. Clin Cancer Res 11: 1842-1848, 2005.

31. Krambeck AE, Thompson RH, Dong H, Lohse CM, Park ES, Kuntz SM, Leibovich BC, Blute ML, Cheville JC and Kwon ED: B7-H4 expression in renal cell carcinoma and tumor vasculature: Associations with cancer progression and survival. Proc Natl Acad Sci USA 103: 10391-10396, 2006.

32. Zang X, Thompson RH, Al-Ahmadie HA, Serio AM, Reuter VE, Eastham JA, Scardino PT, Sharma P and Allison JP: B7-H3 and $\mathrm{B} 7 \mathrm{x}$ are highly expressed in human prostate cancer and associated with disease spread and poor outcome. Proc Natl Acad Sci USA 104: 19458-19463, 2007.

33. Shi H, Ji M, Wu J, Zhou Q, Li X, Li Z, Zheng X, Xu B, Zhao W, Wu C and Jiang J: Serum B7-H4 expression is a significant prognostic indicator for patients with gastric cancer. World J Surg Oncol 12: 188, 2014.

34. Simon I, Zhuo S, Corral L, Diamandis EP, Sarno MJ, Wolfert RL and Kim NW: B7-h4 is a novel membrane-bound protein and a candidate serum and tissue biomarker for ovarian cancer. Cancer Res 66: 1570-1575, 2006.

35. Zhang C, Li Y and Wang Y: Diagnostic value of serum B7-H4 for hepatocellular carcinoma. J Surg Res 197: 301-306, 2015. 